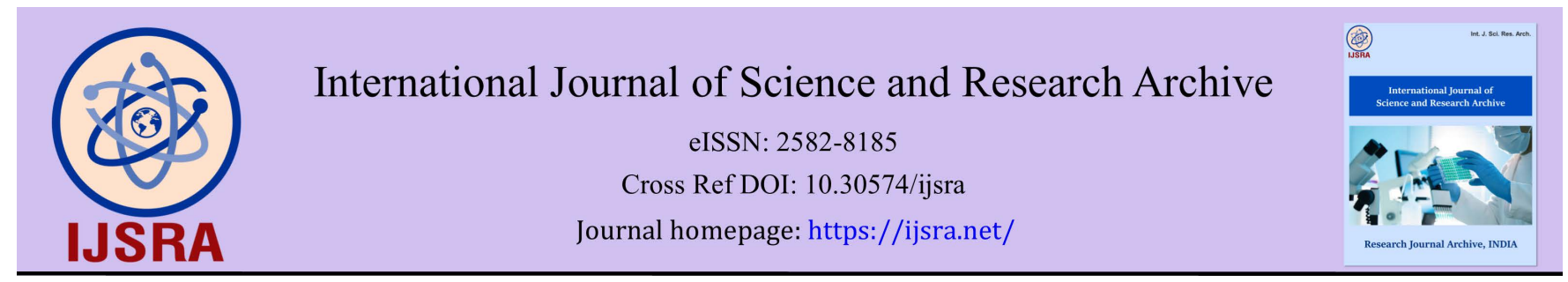

(RESEARCH ARTICLE)

\title{
Challenge and hope for parents who have cancer
}

\author{
Yuko Akagawa ${ }^{1, *}$, Sachiko Makabe ${ }^{1}$, Tomoko Ito ${ }^{1}$, Yutaka Kimura ${ }^{2}$ and Hideaki Andoh ${ }^{1,3}$ \\ ${ }^{1}$ Department of Nursing, Akita University Graduate School of Health Sciences, Akita, Japan. \\ 2 Faculty of Systems Science and Technology, Akita Prefectural University, Japan. \\ 3 Palliative care center, Akita University Hospital, Akita, Japan.
}

International Journal of Science and Research Archive, 2021, 03(01), 136-147

Publication history: Received on 20 July 2021; revised on 24 August 2021; accepted on 26 August 2021

Article DOI: https://doi.org/10.30574/ijsra.2021.3.1.0125

\begin{abstract}
Parents who have cancer face particular problems in their relationships with children. This study aims to clarify 1) the current status of challenge/hope in parental cancer, 2) the factors related to challenge/hope, and 3) the relationship between challenge/hope and QOL/stress-coping ability. Cross-sectional national survey was conducted at designated cancer hospitals in Japan. Participants were undergoing cancer treatment and have children under 18 years old. The questionnaire included demographic data, QOL, ability to cope with stress, and challenge/hope. From 11 hospitals, 54 patients (response rate: 79.4\%) participated. Majority of participants were female (72.2\%) with the mean age of $39.3 \pm$ 5.3 (SD). The total score was QOL (FACT-G: $50.4 \pm 16.2$ ), stress-coping ability (SOC: $46.7 \pm 10.4$ ). The main challenges were an inability to fulfill the parental role and children's mental suffering due to loneliness. The main aspects of hope were the value of the children's present self, being a parent, and strengthening family bonds. Gender and disease duration were significantly related with challenge/hope. Challenge was significantly related with QOL/stress-coping ability. Parents who have cancer derive hope from their relationship with their children, although they feel a gap between their ideal role/value as a parent and their current status.
\end{abstract}

Keywords: Cancer; Parental cancer; Challenge; Hope; Children

\section{Introduction}

Parents who have cancer face particular problems in their relationships with their children [1]. Parents experience changes in their lives, environment, and parental roles due to cancer treatment. Additionally, parents suffer losses and pain related to parenting. The cancer treatment tends to be aggressive, and has an effect on the spirit, mind and body of the patient. These circumstances largely impact diagnosed parents' children and may cause psychological and social problems. For parents to be hospitalized and treated smoothly, it is important to perform family care that includes the children at the same time as patient care. On the other hand, parental responsibilities and feelings about their child may support the treatment, and it is assumed that the child's existence is source of hope for the parent. Nevertheless, most recent papers focus only on challenge not on hope.

A literature review [2], identifies two aspects, challenge and hope, in parents who have cancer. The challenge contents of parents who suffer from cancer include "role restriction due to physical problems," "psychological strain," "social effort," and "concern about the future of their children." Hope includes "children's spiritual support and encouragement," "meaning as parents," "child growth", and "positive changes in family relationships." Challenge and hope in parents vary according to individual backgrounds, types of treatment, quality of life (QOL), and the ability to cope with stress etc. [3]. From this review, a theoretical framework can be developed. Nevertheless, this theory needs to be tested to understand these populations better.

\footnotetext{
* Corresponding author: Yuko Akagawa

Department of Nursing, Akita University Graduate School of Health Sciences, Akita, Japan.
}

Copyright (c) 2021 Author(s) retain the copyright of this article. This article is published under the terms of the Creative Commons Attribution Liscense 4.0. 
In Japan, patients who have children under the age of 18 are about 56,000 per year and a total of about 87,000 children [4]. Although reports from Asia are limited, the number of patient's aged between 20 to 50 years old is increasing partly because couples are marrying later. The global burden of cancer continues to increase largely in global, and changes in the pattern of cancer [5]. Understanding the current situation related to the emotions and roles of parents can help to improve the QOL of parents as well as children. This study aims to clarify 1) the current status of challenge/hope in parental cancer, 2) the factors related to challenge/hope, and 3) the relationship between challenge/hope and QOL/stress-coping ability.

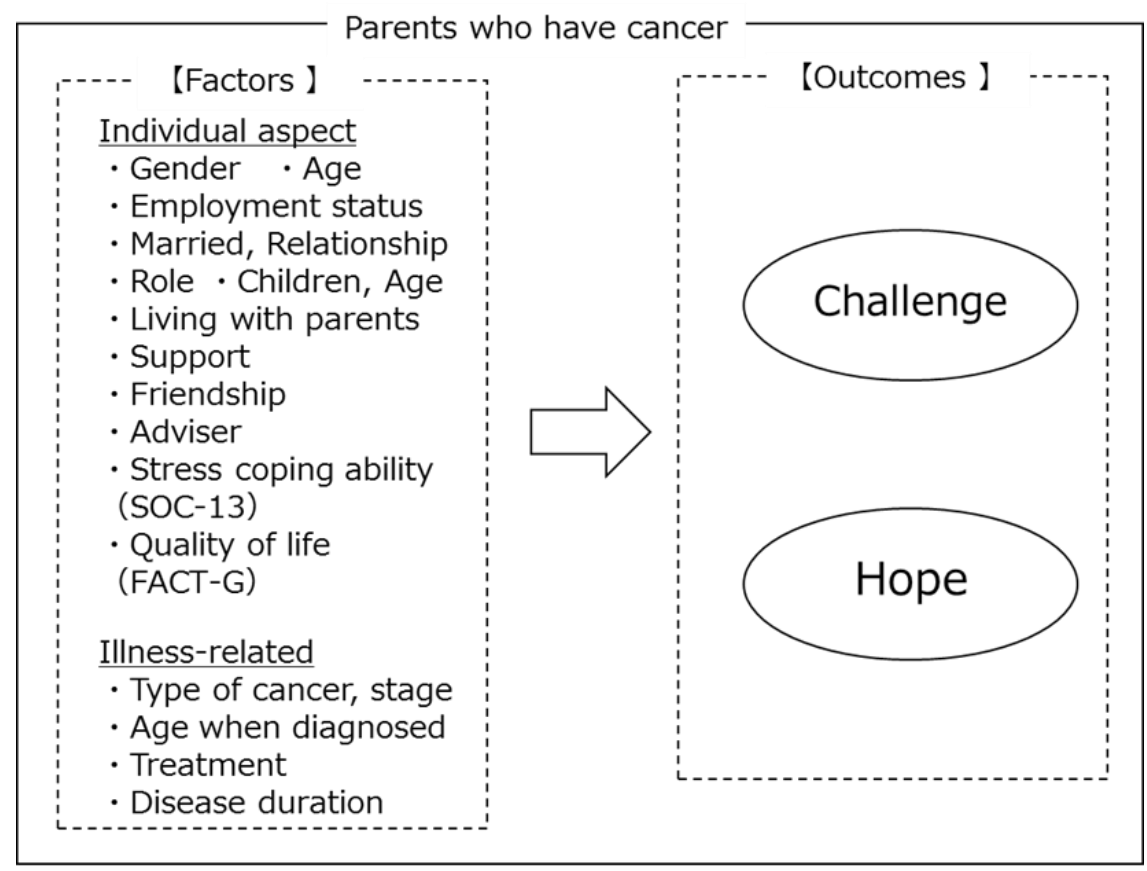

Figure 1 Conceptual diagram

\subsection{Definition of operational terms}

Challenge is defined by parents' struggle in the sense of values and an inability to look after their children. Parents' spiritual pain is reduced by strengthening their relationship with their children. Hope is what strengthens parents' relationship with their children. Challenge and hope develop from the relationship parents have with their children while undergoing cancer treatment. Cancer treatment continues over a long period. The side effects of treatment and anxiety of recurrence has an impact on the parental role.

\section{Methods}

\subsection{Study design}

Cross-sectional national survey in Japan

\subsection{Participants}

Patients who were undergoing cancer treatment at designated cancer hospitals and have children under 18 years of age. There were no inclusion criteria, exclusion criteria were those with mental illness.

\subsection{Data collection and questionnaires}

The survey period was between April and September 2018. A letter containing the aim of the study and a sample of the questionnaire, were sent to all designated cancer hospitals in Japan.

The contents of the questionnaire included personal information, illness-related information, and questions related to challenge and hope (Fig. 1). Individual information included personal attributes such as gender, age, employment status, marriage status, status of living with parents, number of children and age, QOL, and ability to cope with stress. Illness- 
related information included the type of cancer, the patient's age when diagnosed, type of treatment, and disease duration.

The Sense of Coherence (SOC) is a health engendering orientation which is a stress-resistance resource to measure stress-coping ability [6]. SOC consists of 13 items that are scored using a seven-point Likert-type scale. Each item is rated from 1 to 7 points, with a maximum total score of 91 points. A high SOC score indicates a higher ability to cope with stress.

The QOL of cancer patients was measured using a Functional Assessment of Cancer Therapy Scale General (FACT-G) developed for cancer patients [7]. It consists of 4 subscales, and 27 items: 7 physical items, 7 social/family items, 6 psychological items, and 7 activity/functional items. Each item is rated from 0 to 4 points on a 5-point Likert-type scale with a maximum score of 108 points: the higher the score, the better the QOL. The use of the FACT-G scale is increasing rapidly internationally, and the reliability and validity of the Japanese version has been established [8]. In order to use these scales, the developer performed a prescribed use registration procedure and obtained permission to use it as well as the SOC scale.

QOL demonstrates the patient's current status when answering the questionnaire. The patient's stress-coping ability is developed over a whole life. Consequently, QOL and stress-coping ability were set as a baseline and embedded into the patient's individual characteristics. Challenge and hope related to children are outcomes of this study. Based on the definitions of challenge and hope, questionnaire items were extracted from current research papers. A literature review [2] helped to back up the theoretical framework. A professional panel included three cancer nursing researchers, a palliative care doctor, and a statistician. Items were checked and revised numerous times until the professional panel reached consensus. For reliability, a partial correlation coefficient was used. Only one item from hope appeared as $\mathrm{r}=$ 0 [9]. Finally, challenge contained 13 items, and hope contained 12 items (Tabe2, 3). Before analyzing the data for challenge/hope, statistically identical items needed to be deleted in order to compare the total score of challenge/hope with the total score of QOL/stress-coping. The answering style was "Strongly agree," "Agree," "Neither," "Disagree," and "Strongly disagree." Each item was rated from 1 to 5 points on a 5-point Likert-type scale, with the total for challenge scored between 13 to 65 points, and for hope between 12 to 60 points. The higher the score indicates higher the challenge/hope.

\subsection{Ethical consideration}

This study was approved by the Ethics Committee of the author's University (Approval No. 1899). When institutions require the approval of an ethics committee, a key person from the institution applies and receives an approval with support from the author. Patients receive a letter informing them of the study's aims and methods, which includes the following: 1) their privacy will be strictly protected; 2 ) their refusal to participate will not influence the care provided; 3) participation is voluntary; 4) data will only be presented in an aggregated form in professional journals; and 5) the disadvantage lay in the time required to respond to the questionnaire. Returning the questionnaire would be regarded as consent to participate in the study.

\subsection{Data analysis}

The questionnaire was included in the analysis if approximately $80 \%$ or more of the questions were answered. Individual and illness aspects were aggregated. Parental background during cancer treatment was summarized, and the total, mean, and standard deviation of stress-coping ability (SOC-13) and QOL (FACT-G) were calculated.

To detect the factors related to challenge/hope, first the answers for challenge and hope were categorized in 2 groups: "Strongly agree" and "Agree" were categorized under "Yes", and "Neither," "Disagree," and "Strongly disagree" were categorized under "No" to avoid statistical errors due to the small sample size. Next, each item for challenge/hope was used as objective variables, and attributes were used as explanatory variables, which were confirmed by either chisquare test or Fisher's exact test with the significance level of $5 \%$.

Furthermore, the total score for challenge and hope were used as an objective variable, and stress-coping ability (SOC13) and QOL (FACT-G) were used as an explanatory variable to confirm how attributes are related. Spearman's rank correlation coefficient was used for statistical analyses, with the significance level of 5\%. The statistical software JMP ${ }^{\circledR 13.0 ~(S A S ~ i n s t i t u t e) ~ w a s ~ u s e d ~ f o r ~ t h e ~ a n a l y s i s . ~}$ 


\section{Results}

After cooperation from 11 designated cancer hospitals (response rate: $2.7 \%$ ), it took about 9 months to receive ethical reviews from seven facilities. An average of five questionnaires were sent to each institution, and 54 patients (response rate: $79.4 \%$ ) participated. All returned questionnaires had an adequate level of answering of over $80 \%$.

Table 1 shows that the majority of participants were female $(72.2 \%)$ with the mean age of $39.3 \pm 5.3$ (SD). The number of children was evenly distributed between one and two, and the mean age of the children was $9.0 \pm 4.5$. The participants also said that the main person they could talk to about any concerns, other than a family member, was a friend. The total score of QOL (FACT-G) was $50.4 \pm 16.2$ and stress-coping ability was $46.7 \pm 10.4$. The main type of cancer was breast cancer (42.5\%), gynecological cancer (22.2\%), and digestive organ cancer (20.4\%). Most of the patients (63.0\%) had been diagnosed more than five years ago at the mean age of $36.4 \pm 5.1$. The most frequent diagnosis was with stage III cancer (53.7\%). Most of current treatment was chemotherapy (78.5\%).

Table 1 Participant's background $\quad \mathrm{N}=54$

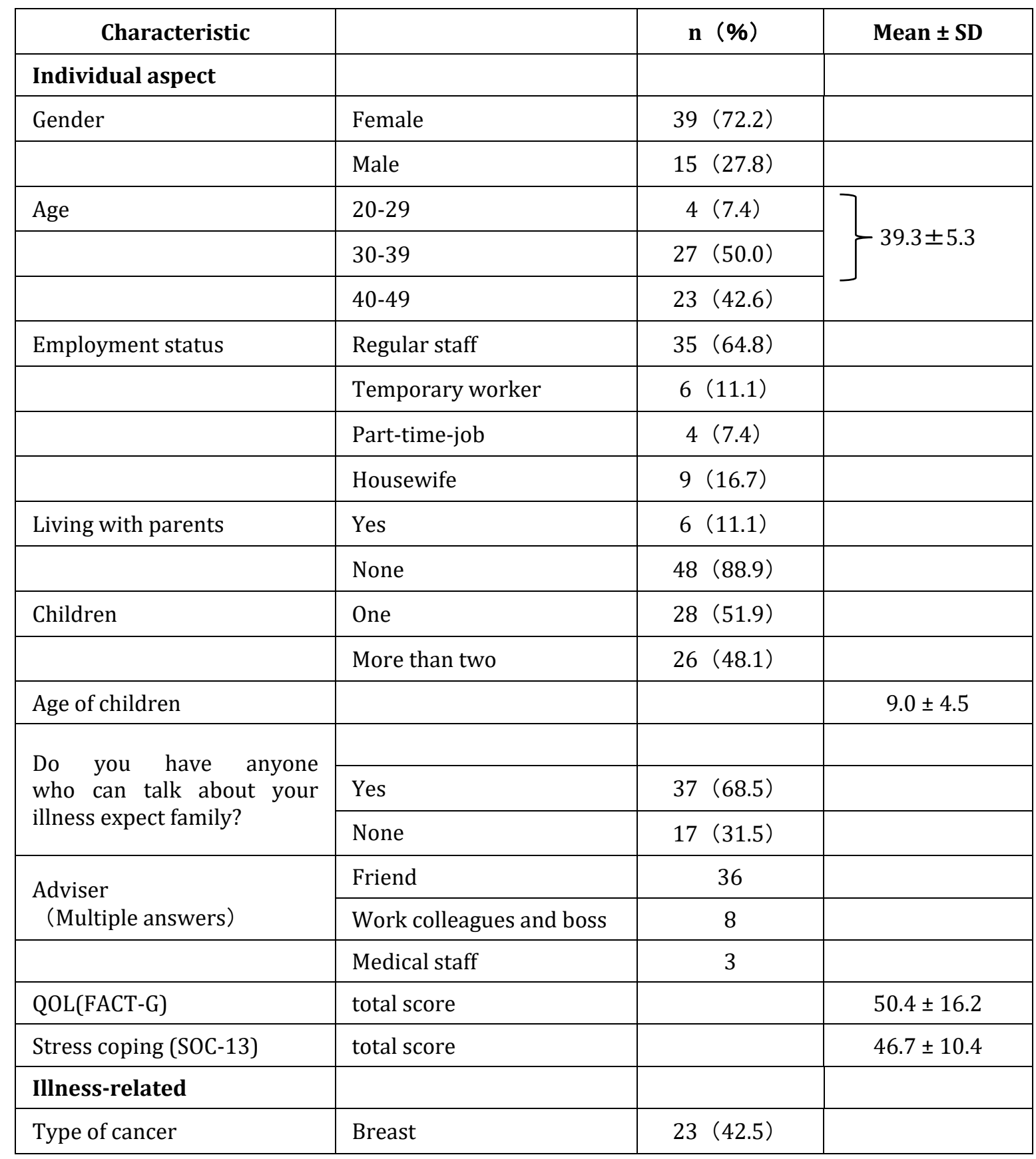




\begin{tabular}{|c|c|c|c|}
\hline & Gynecology & $12(22.2)$ & \\
\hline & Digestive organ & $11(20.4)$ & \\
\hline & Lung & $3(5.6)$ & \\
\hline & Testis & $2(3.7)$ & \\
\hline & Other & $3(5.6)$ & \\
\hline Age when diagnosed & & & $36.4 \pm 5.1$ \\
\hline \multirow[t]{2}{*}{ Disease duration } & Less than 5 years & $20(37.0)$ & \multirow{2}{*}{$3.2 \pm 3.2$} \\
\hline & 5 years or more & $34(63.0)$ & \\
\hline \multirow[t]{5}{*}{ Stage } & I & $2(3.7)$ & \\
\hline & II & $18(33.3)$ & \\
\hline & III & $29(53.7)$ & \\
\hline & IV & $4(7.4)$ & \\
\hline & No explanation & $1(1.9)$ & \\
\hline Treatment & Surgery & $0(0.0)$ & \\
\hline \multirow[t]{4}{*}{ (Multiple answers) } & Chemical treatment & 44（78.5） & \\
\hline & Radiation therapy & $2(3.6)$ & \\
\hline & Hormonal therapy & $7(12.5)$ & \\
\hline & Follow-up & $3(5.4)$ & \\
\hline
\end{tabular}

For the distribution of challenge, six items were answered more than $80.0 \%$ of the time as "agree". In order of highest score, "I did not want to have children mentally hurt by my illness (94.3\%)," "I was afraid my children might suffer mentally in the future. (90.6\%)," "I could not play my parental role in full due to physical side effects of treatment (86.8\%)," "I worried whether children might feel lonely (83.0\%)," "I could not attend some important events of my children due to my medical treatment (83.0\%)," and "I was sometimes afraid that children might feel inconvenience in everyday life (81.1\%)." For the distribution of hope, six items were answered more than $80.0 \%$ of the time as "agree". In order of the highest score, "I face the stressful treatment for my children (92.4\%)," "I am encouraged by an awareness of being a parent (88.7\%)," "I value the time with my family (84.9\%)," "My presence is required as a parent (81.1\%)," and "Familial bonds became strengthened (81.1\%)."

Table 2 presented the relationship between challenge and participants' background. Male parents were significantly aligned with "reprimanding the children too often," "feeling upset," and "worrying about economic problems." Women were significantly aligned with "worrying about heredity." The absence of an adviser was significantly related with "not able to play my parental role," "reprimanding children too often," "feeling upset," and "worrying about economic problems." The diagnosis period of 5 years or more was significantly related with "worrying about economic problems."

Table 3 presented the relationship between hope and participants' background. Male was significantly aligned with "improving of parental role," "value the time with the family," and "familial bonds became strengthened." Women was significantly related with "required as a parent," and "children begin to help with the housework." Having an adviser was significantly aligned with "required as a parent." and "to become kind and gentle." The diagnosis period of less than 5 years was significantly related with "increasing number of opportunities to talk with children." Stages was significantly related with "children become a kind of adviser," and "improving my relationship with the children." There were no significant differences between ages and challenge/hope. 
Table 2 Relationship between challenge and participant's background $\quad N=53$

\begin{tabular}{|c|c|c|c|c|c|c|c|c|c|c|c|c|c|c|c|c|}
\hline \multirow[b]{2}{*}{ Challenge } & & \multicolumn{3}{|l|}{ Gender } & \multicolumn{3}{|l|}{ Age } & \multicolumn{3}{|c|}{ Adviser ${ }^{-}$} & \multicolumn{3}{|c|}{ Disease duration } & \multicolumn{3}{|l|}{ Stage } \\
\hline & & $\begin{array}{l}\text { Male } \\
(n=14)\end{array}$ & $\begin{array}{l}\text { Female } \\
(n=39)\end{array}$ & $p$ & $\begin{array}{l}20-39 \\
(n=31)\end{array}$ & $\begin{array}{l}40-49 \\
(n=22)\end{array}$ & $p$ & $\begin{array}{l}\text { Yes } \\
(n=37)\end{array}$ & $\begin{array}{l}\text { None } \\
(n=16)\end{array}$ & $p$ & $\begin{array}{l}\text { Less } \\
\text { than } 5 \\
\text { years } \\
(n=20)\end{array}$ & \begin{tabular}{|l|}
5 \\
years \\
or \\
more \\
$(n=33)$ \\
\end{tabular} & $p$ & $\begin{array}{l}I \cdot I I \\
(n=19)\end{array}$ & $\begin{array}{l}\text { III } \\
\text { IV } \\
(n=33)\end{array}$ & $p$ \\
\hline \multirow{2}{*}{$\begin{array}{l}\text { I could not play my } \\
\text { parental role in full } \\
\text { due to physical side } \\
\text { effects of treatment. }\end{array}$} & $\begin{array}{c}\text { Yes } \\
(n=45)\end{array}$ & $10(71)$ & $35(90)$ & \multirow{2}{*}{0.1} & $27(87)$ & $18(82)$ & \multirow{2}{*}{0.6} & $34(92)$ & 11(69) & \multirow{2}{*}{$\stackrel{*}{*}+0.03$} & $19(95)$ & $26(79)$ & \multirow{2}{*}{0.11} & $15(79)$ & $29(87)$ & \multirow{2}{*}{0.39} \\
\hline & $\begin{array}{c}\text { No } \\
(\mathrm{n}=8)\end{array}$ & $4(29)$ & $4(10)$ & & $4(13)$ & $4(18)$ & & $3(8)$ & $5(31)$ & & $1(5)$ & $7(21)$ & & $4(21)$ & $4(13)$ & \\
\hline \multirow{2}{*}{$\begin{array}{l}\text { I could not attend } \\
\text { some important } \\
\text { events of } \text { my } \\
\text { children due to my } \\
\text { medical treatment. }\end{array}$} & $\begin{array}{c}\text { Yes } \\
(n=43)\end{array}$ & $9(64)$ & $34(87)$ & \multirow[b]{2}{*}{0.6} & $26(84)$ & $17(77)$ & \multirow[b]{2}{*}{0.5} & $32(86)$ & $11(69)$ & \multirow[b]{2}{*}{0.12} & $17(85)$ & $26(79)$ & \multirow[b]{2}{*}{0.58} & $15(79)$ & $27(82)$ & \multirow[b]{2}{*}{0.8} \\
\hline & $\begin{array}{c}\text { No } \\
(n=10)\end{array}$ & $5(36)$ & $5(13)$ & & $5(16)$ & $5(23)$ & & $5(14)$ & $5(31)$ & & $3(15)$ & $7(21)$ & & $4(21)$ & $6(18)$ & \\
\hline \multirow{2}{*}{$\begin{array}{l}\text { I felt sorry for being } \\
\text { unable to take care of } \\
\text { my children. }\end{array}$} & $\begin{array}{c}\text { Yes } \\
(n=41)\end{array}$ & 11(79) & $30(77)$ & \multirow{2}{*}{0.9} & $26(84)$ & $15(68)$ & \multirow{2}{*}{0.2} & 29(78) & $12(75)$ & \multirow{2}{*}{0.79} & $14(70)$ & $27(82)$ & \multirow{2}{*}{0.32} & $14(75)$ & $26(79)$ & \multirow{2}{*}{0.67} \\
\hline & $\begin{array}{c}\text { No } \\
(n=12)\end{array}$ & $3(21)$ & $9(23)$ & & $5(16)$ & $7(32)$ & & $8(22)$ & $4(25)$ & & $6(30)$ & $6(18)$ & & $5(25)$ & $7(11)$ & \\
\hline \multirow{2}{*}{$\begin{array}{l}\text { I felt it difficult to } \\
\text { communicate with } \\
\text { my children when I } \\
\text { was in bad condition. }\end{array}$} & $\begin{array}{c}\text { Yes } \\
(n=36)\end{array}$ & $12(86)$ & $24(62)$ & \multirow{2}{*}{0.1} & $22(71)$ & $14(64)$ & \multirow{2}{*}{0.6} & 23(63) & $13(81)$ & \multirow{2}{*}{0.17} & $12(60)$ & $24(73)$ & \multirow{2}{*}{0.34} & $15(79)$ & $20(61)$ & \multirow{2}{*}{0.17} \\
\hline & $\begin{array}{c}\text { No } \\
(n=17)\end{array}$ & $2(14)$ & $15(38)$ & & $9(29)$ & $8(36)$ & & $14(37)$ & $3(19)$ & & $8(40)$ & $9(17)$ & & $4(21)$ & 13(39) & \\
\hline \multirow{2}{*}{$\begin{array}{l}\text { I did not want to } \\
\text { have children } \\
\text { mentally hurt by my } \\
\text { illness. }\end{array}$} & $\begin{array}{c}\text { Yes } \\
(n=51)\end{array}$ & $13(93)$ & $38(97)$ & \multirow{2}{*}{$\mathrm{N} / \mathrm{A}$} & $31(100)$ & $20(91)$ & \multirow{2}{*}{ N/A } & $36(97)$ & $15(94)$ & & $20(100)$ & $31(94)$ & & $19(100)$ & $32(97)$ & \\
\hline & $\begin{array}{c}\text { No } \\
(\mathrm{n}=2)\end{array}$ & $1(7)$ & $1(3)$ & & $0(0)$ & $2(9)$ & & $1(3)$ & $1(6)$ & N/A & $0(0)$ & $2(6)$ & $\mathrm{N} / \mathrm{A}$ & $0(0)$ & $1(3)$ & N/A \\
\hline I worried whether & $\begin{array}{c}\text { Yes } \\
(n=45)\end{array}$ & 11(79) & $34(87)$ & & $28(90)$ & $17(77)$ & & $32(86)$ & $13(81)$ & & $17(85)$ & $28(85)$ & & $17(89)$ & $28(85)$ & \\
\hline $\begin{array}{l}\text { children might feel } \\
\text { lonely. }\end{array}$ & $\begin{array}{c}\text { No } \\
(n=8)\end{array}$ & $3(21)$ & $5(13)$ & 0.4 & $3(10)$ & $5(23)$ & 0.2 & $5(14)$ & $3(19)$ & 0.6 & $3(15)$ & $5(15)$ & 0.99 & $2(11)$ & $5(15)$ & 0.64 \\
\hline $\begin{array}{l}\text { I felt depressed to } \\
\text { think that children }\end{array}$ & $\begin{array}{c}\text { Yes } \\
(n=41)\end{array}$ & 11(79) & $30(77)$ & 0.9 & $25(81)$ & $16(73)$ & 0.5 & $30(81)$ & $11(69)$ & 0.32 & $18(90)$ & $23(70)$ & 0.09 & $15(79)$ & $26(79)$ & 0.99 \\
\hline
\end{tabular}


International Journal of Science and Research Archive, 2021, 03(01), 136-147

\begin{tabular}{|c|c|c|c|c|c|c|c|c|c|c|c|c|c|c|c|c|}
\hline $\begin{array}{l}\text { might be holding } \\
\text { down what they } \\
\text { wanted to say. }\end{array}$ & $\begin{array}{c}\text { No } \\
(n=12)\end{array}$ & $3(21)$ & $9(23)$ & & $6(19)$ & $6(21)$ & & $7(19)$ & $5(31)$ & & $2(10)$ & $10(30)$ & & $4(21)$ & $7(11)$ & \\
\hline \multirow{2}{*}{$\begin{array}{l}\text { I reprimanded } \\
\text { children too often. }\end{array}$} & $\begin{array}{c}\text { Yes } \\
(n=20)\end{array}$ & $14(100)$ & $6(15)$ & \multirow{2}{*}{$\begin{array}{c}* * \\
0.0001\end{array}$} & 11(35) & $9(41)$ & \multirow{2}{*}{0.7} & $6(16)$ & $14(88)$ & \multirow{2}{*}{$\stackrel{* *}{* *}$} & $9(45)$ & 11(33) & \multirow{2}{*}{0.4} & $8(42)$ & $12(36)$ & \multirow{2}{*}{0.68} \\
\hline & $\begin{array}{c}\text { No } \\
(n=33)\end{array}$ & $0(0)$ & $33(75)$ & & $20(65)$ & $13(59$ & & $31(84)$ & $2(12)$ & & 11(55) & $22(67)$ & & $11(58)$ & $21(64)$ & \\
\hline \multirow{2}{*}{$\begin{array}{l}\text { I felt like that I was } \\
\text { upset by my } \\
\text { children. }\end{array}$} & $\begin{array}{c}\text { Yes } \\
(n=17)\end{array}$ & 13(93) & $4(10)$ & \multirow{2}{*}{$\begin{array}{c}* * \\
0.0001\end{array}$} & $9(29)$ & $8(36)$ & \multirow{2}{*}{0.6} & $5(14)$ & $12(75)$ & \multirow{2}{*}{$\stackrel{* *}{* *}$} & $7(35)$ & $10(30)$ & \multirow{2}{*}{0.72} & $7(37)$ & $10(30)$ & \multirow{2}{*}{0.63} \\
\hline & $\begin{array}{c}\text { No } \\
(n=36)\end{array}$ & $1(7)$ & $35(90)$ & & $22(71)$ & $14(64)$ & & $32(86)$ & $4(25)$ & & $13(65)$ & $23(70)$ & & $12(63)$ & $23(70)$ & \\
\hline \multirow{2}{*}{$\begin{array}{l}\text { I was sometimes } \\
\text { afraid that children } \\
\text { might } \\
\text { inconvenience in } \\
\text { everyday life. }\end{array}$} & $\begin{array}{c}\text { Yes } \\
(n=45)\end{array}$ & 13(93) & $32(82)$ & \multirow[b]{2}{*}{0.3} & $26(84)$ & $19(86)$ & \multirow[b]{2}{*}{0.8} & $30(81)$ & $15(94)$ & \multirow[b]{2}{*}{0.24} & $17(85)$ & $28(85)$ & \multirow[b]{2}{*}{0.99} & $17(89)$ & $28(85)$ & \multirow[b]{2}{*}{0.64} \\
\hline & $\begin{array}{c}\text { No } \\
(n=8)\end{array}$ & $1(7)$ & $7(18)$ & & $5(16)$ & $4(18)$ & & $7(19)$ & $1(6)$ & & $3(15)$ & $5(15)$ & & $2(11)$ & $5(15)$ & \\
\hline \multirow{2}{*}{$\begin{array}{l}\text { I was worried that } \\
\text { economic problems } \\
\text { might affect children. }\end{array}$} & $\begin{array}{c}\text { Yes } \\
(n=38)\end{array}$ & $14(100)$ & $24(62)$ & \multirow{2}{*}{$\begin{array}{c}* * \\
0.006\end{array}$} & $20(65)$ & 18(82) & \multirow{2}{*}{0.2} & $22(59)$ & $16(100)$ & \multirow{2}{*}{$\begin{array}{c}* * \\
0.0003\end{array}$} & 11(55) & $27(82)$ & \multirow{2}{*}{$\begin{array}{c}* \\
0.04\end{array}$} & $14(75)$ & $23(70)$ & \multirow{2}{*}{0.76} \\
\hline & $\begin{array}{c}\text { No } \\
(n=15)\end{array}$ & $0(0)$ & $15(38)$ & & $11(35)$ & $4(18)$ & & $15(41)$ & $0(0)$ & & $9(45)$ & $6(18)$ & & $5(25)$ & $10(30)$ & \\
\hline \multirow{2}{*}{$\begin{array}{l}\text { I was afraid my } \\
\text { children might suffer } \\
\text { mentally in the } \\
\text { future. }\end{array}$} & $\begin{array}{c}\text { Yes } \\
(n=49)\end{array}$ & $12(86)$ & $37(95)$ & \multirow{2}{*}{0.27} & $29(94)$ & $20(91)$ & \multirow{2}{*}{$\mathrm{N} / \mathrm{A}$} & $35(95)$ & $14(88)$ & \multirow{2}{*}{$\mathrm{N} / \mathrm{A}$} & $19(95)$ & $30(91)$ & \multirow{2}{*}{$\mathrm{N} / \mathrm{A}$} & $18(95)$ & $30(91)$ & \multirow{2}{*}{$\mathrm{N} / \mathrm{A}$} \\
\hline & $\begin{array}{c}\text { No } \\
(\mathrm{n}=4)\end{array}$ & $2(14)$ & $2(5)$ & & $2(6)$ & $2(9)$ & & $2(5)$ & $2(12)$ & & $1(5)$ & $3(9)$ & & $1(5)$ & $3(9)$ & \\
\hline \multirow{2}{*}{$\begin{array}{l}\text { I felt sorry that } \\
\text { genetic cancer might } \\
\text { be passed down. }\end{array}$} & $\begin{array}{c}\text { Yes } \\
(n=28)\end{array}$ & $3(21)$ & $25(64)$ & \multirow{2}{*}{$\begin{array}{c}* * \\
0.006\end{array}$} & $16(51)$ & $12(55)$ & \multirow{2}{*}{0.8} & $23(62)$ & $5(31)$ & \multirow{2}{*}{$\begin{array}{c}* \\
0.04\end{array}$} & $10(50)$ & $18(55)$ & & $9(47)$ & $18(55)$ & \\
\hline & $\begin{array}{l}\text { No } \\
(n=25)\end{array}$ & $11(79)$ & $14(36)$ & & $15(49)$ & $10(45)$ & & $14(25)$ & $11(69)$ & & $10(50)$ & $15(45)$ & 0.15 & $10(53)$ & $15(45)$ & 0.02 \\
\hline
\end{tabular}


Table 3 Relationship between hope and participant's background $\quad N=53$

\begin{tabular}{|c|c|c|c|c|c|c|c|c|c|c|c|c|c|c|c|c|}
\hline \multirow[b]{2}{*}{ Hope } & & \multicolumn{3}{|l|}{ Gender } & \multicolumn{3}{|l|}{ Age } & \multicolumn{3}{|c|}{ Adviser ${ }^{-}$} & \multicolumn{3}{|c|}{ Disease duration } & \multicolumn{3}{|l|}{ Stage } \\
\hline & & $\begin{array}{l}\text { Male } \\
(n=14)\end{array}$ & $\begin{array}{l}\text { Female } \\
(n=39)\end{array}$ & $p$ & $\begin{array}{l}20-39 \\
(n=31)\end{array}$ & $\begin{array}{l}40-49 \\
(n=22)\end{array}$ & $p$ & $\begin{array}{l}\text { Yes } \\
(n=37)\end{array}$ & $\begin{array}{l}\text { None } \\
(n=16)\end{array}$ & $p$ & $\begin{array}{l}\text { Less } \\
\text { than } 5 \\
\text { years } \\
(n=20)\end{array}$ & $\begin{array}{l}5 \\
\text { years } \\
\text { or } \\
\text { more } \\
(n=33)\end{array}$ & $p$ & $\begin{array}{l}I \cdot I \\
(n=19)\end{array}$ & $\begin{array}{l}\text { III } \cdot \text { IV } \\
(n=33)\end{array}$ & $p$ \\
\hline \multirow{2}{*}{$\begin{array}{l}\text { I am } \\
\text { encouraged to } \\
\text { see children } \\
\text { enjoying life } \\
\text { cheerfully. }\end{array}$} & Yes $(n=50)$ & $13(93)$ & $37(97)$ & \multirow[b]{2}{*}{$\mathrm{N} / \mathrm{A}$} & $31(100)$ & $19(90)$ & \multirow[b]{2}{*}{$\mathrm{N} / \mathrm{A}$} & $35(97)$ & $15(94)$ & \multirow[b]{2}{*}{$\mathrm{N} / \mathrm{A}$} & $20(100)$ & $30(94)$ & \multirow[b]{2}{*}{ N/A } & $18(95)$ & $31(97)$ & \multirow[b]{2}{*}{ N/A } \\
\hline & No $(n=2)$ & $1(7)$ & $2(3)$ & & $0(0)$ & $3(10)$ & & $2(3)$ & $1(6)$ & & $0(0)$ & $3(6)$ & & $1(5)$ & $2(3)$ & \\
\hline \multirow{2}{*}{$\begin{array}{l}\text { I face a } \\
\text { stressfull } \\
\text { treatment for } \\
\text { my children. }\end{array}$} & Yes $(n=50)$ & $14(100)$ & $36(95)$ & \multirow[b]{2}{*}{$\mathrm{N} / \mathrm{A}$} & $29(94)$ & $22(100)$ & \multirow[b]{2}{*}{$\mathrm{N} / \mathrm{A}$} & $19(51)$ & $15(94)$ & \multirow[b]{2}{*}{$\mathrm{N} / \mathrm{A}$} & $20(100)$ & $30(94)$ & \multirow[b]{2}{*}{$\mathrm{N} / \mathrm{A}$} & $18(95)$ & $23(69)$ & \multirow[b]{2}{*}{$\mathrm{N} / \mathrm{A}$} \\
\hline & No $(n=2)$ & $0(0)$ & $3(59)$ & & $2(6)$ & $0(0)$ & & $18(49)$ & $1(6)$ & & $0(0)$ & $3(6)$ & & $1(5)$ & $10(31)$ & \\
\hline \multirow{2}{*}{$\begin{array}{l}\text { I am } \\
\text { encouraged by } \\
\text { an awareness } \\
\text { of being a } \\
\text { parent. }\end{array}$} & Yes $(n=49)$ & $13(93)$ & $36(95)$ & \multirow{2}{*}{$\mathrm{N} / \mathrm{A}$} & $30(97)$ & $19(90)$ & \multirow{2}{*}{$\mathrm{N} / \mathrm{A}$} & $35(97)$ & $14(87)$ & \multirow{2}{*}{0.17} & $\begin{array}{l}19 \\
(95)\end{array}$ & $30(94)$ & \multirow{2}{*}{$\mathrm{N} / \mathrm{A}$} & $17(89)$ & $31(97)$ & \multirow{2}{*}{0.28} \\
\hline & No $(n=3)$ & $1(7)$ & $3(59)$ & & $1(3)$ & $3(10)$ & & $2(3)$ & $2(13)$ & & $1(5)$ & $3(6)$ & & $2(11)$ & $2(3)$ & \\
\hline \multirow{2}{*}{$\begin{array}{l}\text { Parental } \\
\text { attitude is } \\
\text { improved } \\
\text { since } \\
\text { diagnosed as } \\
\text { cancer. }\end{array}$} & Yes $(n=30)$ & $12(86)$ & $18(47)$ & \multirow{2}{*}{$\begin{array}{l}* \\
0.01\end{array}$} & $18(58)$ & $12(57)$ & \multirow[b]{2}{*}{0.95} & $19(51)$ & $11(69)$ & \multirow[b]{2}{*}{0.28} & $\begin{array}{l}9 \\
(45)\end{array}$ & $21(66)$ & \multirow[b]{2}{*}{0.14} & $9(47)$ & $21(66)$ & \multirow[b]{2}{*}{0.2} \\
\hline & No $(n=22)$ & $2(14)$ & $21(53)$ & & $13(42)$ & $10(43)$ & & $18(49)$ & $5(31)$ & & $11(58)$ & $12(34)$ & & $10(53)$ & $12(34)$ & \\
\hline \multirow{3}{*}{$\begin{array}{l}\text { My presence is } \\
\text { required as a } \\
\text { parent. }\end{array}$} & Yes $(n=42)$ & $8(57)$ & $34(89)$ & \multirow{2}{*}{$\begin{array}{l}* * \\
0.009\end{array}$} & $24(77)$ & $18(86)$ & \multirow[t]{2}{*}{0.46} & $32(89)$ & $10(63)$ & \multirow{2}{*}{$\begin{array}{l}* \\
0.03\end{array}$} & $\begin{array}{l}17 \\
(85)\end{array}$ & $25(78)$ & 0.54 & $13(68)$ & $28(88)$ & 0.1 \\
\hline & No $(n=10)$ & $6(43)$ & $5(11)$ & & $7(23)$ & $4(14)$ & & $5(11)$ & $6(37)$ & & $3(15)$ & $8(22)$ & & $6(32)$ & $5(12)$ & \\
\hline & $\begin{array}{l}\text { Yes } \\
(n=21)\end{array}$ & $3(21)$ & $18(49)$ & 0.08 & $13(42)$ & $8(40)$ & 0.89 & 18(49) & $3(19)$ & $\begin{array}{l}* \\
0.03\end{array}$ & $\begin{array}{l}11 \\
(58)\end{array}$ & $10(31)$ & 0.06 & $10(53)$ & 19(32) & 0.15 \\
\hline
\end{tabular}


International Journal of Science and Research Archive, 2021, 03(01), 136-147

\begin{tabular}{|c|c|c|c|c|c|c|c|c|c|c|c|c|c|c|c|c|}
\hline $\begin{array}{l}\text { Children } \\
\text { become kind } \\
\text { and gentle. }\end{array}$ & No $(n=30)$ & $11(79)$ & $21(53)$ & & $18(58)$ & $14(60)$ & & $19(51)$ & $13(81)$ & & $9(45)$ & $23(69)$ & & $9(47)$ & $13(68)$ & \\
\hline \multirow{2}{*}{$\begin{array}{l}\text { Children begin } \\
\text { to help with } \\
\text { my } \\
\text { housework. }\end{array}$} & Yes $(n=16)$ & $1(7)$ & $15(39)$ & \multirow{2}{*}{$\begin{array}{l}* \\
0.03\end{array}$} & $10(32)$ & $6(29)$ & \multirow[b]{2}{*}{0.78} & $14(38)$ & $2(13)$ & \multirow[b]{2}{*}{0.06} & $10(50)$ & $6(19)$ & \multirow[b]{2}{*}{$\begin{array}{l}* \\
0.02\end{array}$} & $9(42)$ & $8(25)$ & \multirow[b]{2}{*}{0.2} \\
\hline & No $(n=36)$ & $13(93)$ & $24(61)$ & & $21(68)$ & $16(71)$ & & $23(62)$ & 14(87) & & $10(50)$ & $27(81)$ & & $11(58)$ & $25(78)$ & \\
\hline \multirow{2}{*}{$\begin{array}{l}\text { Children } \\
\text { become a kind } \\
\text { of adviser for } \\
\text { me. }\end{array}$} & Yes $(n=11)$ & $2(14)$ & $9(24)$ & \multirow{2}{*}{0.46} & $5(16)$ & $6(29)$ & \multirow{2}{*}{0.28} & $10(27)$ & $1(6)$ & \multirow{2}{*}{0.08} & $\begin{array}{l}7 \\
\quad(35)\end{array}$ & $4(13)$ & \multirow{2}{*}{0.53} & $7(37)$ & $4(13)$ & \multirow{2}{*}{$\begin{array}{l}* \\
0.04\end{array}$} \\
\hline & No $(n=41)$ & $12(86)$ & $30(79)$ & & $26(84)$ & $16(71)$ & & $27(73)$ & $15(94)$ & & $13(65)$ & $29(87)$ & & $12(63)$ & $29(87)$ & \\
\hline \multirow{2}{*}{$\begin{array}{l}\text { I have an } \\
\text { increasing } \\
\text { number of } \\
\text { opportunities } \\
\text { to talk with } \\
\text { children. }\end{array}$} & Yes $(n=35)$ & $9(64)$ & $\begin{array}{l}26 \\
\quad(68)\end{array}$ & \multirow[b]{2}{*}{0.78} & $18(58)$ & $17(81)$ & \multirow[b]{2}{*}{0.08} & $27(73)$ & $8(50)$ & \multirow[b]{2}{*}{0.08} & 17 & $18(56)$ & \multirow[b]{2}{*}{$\begin{array}{l}* \\
0.03\end{array}$} & $\begin{array}{l}16 \\
(84)\end{array}$ & $19(58)$ & \multirow[b]{2}{*}{0.06} \\
\hline & No $(n=17)$ & $5(36)$ & $13(32)$ & & $13(42)$ & $5(19)$ & & $10(27)$ & $8(50)$ & & $3(15)$ & $15(44)$ & & $3(16)$ & $14(42)$ & \\
\hline \multirow{2}{*}{$\begin{array}{l}\text { Relationship } \\
\text { with children } \\
\text { is improved. }\end{array}$} & Yes $(n=28)$ & $9(64)$ & $\begin{array}{l}19 \\
(50)\end{array}$ & \multirow[t]{2}{*}{0.36} & $16(52)$ & $12(57)$ & \multirow[t]{2}{*}{0.69} & $18(49)$ & $19(63)$ & \multirow[t]{2}{*}{0.4} & $\begin{array}{l}13 \\
\quad(25)\end{array}$ & $15(47)$ & \multirow[t]{2}{*}{0.2} & $\begin{array}{l}14 \\
\quad(74)\end{array}$ & $14(42)$ & \multirow{2}{*}{$\begin{array}{l}* \\
0.04\end{array}$} \\
\hline & No $(n=24)$ & $5(36)$ & $20(50)$ & & $15(48)$ & $10(43)$ & & $19(51)$ & $6(37)$ & & $7(75)$ & $18(56)$ & & $5(26)$ & $19(58)$ & \\
\hline \multirow{2}{*}{$\begin{array}{l}\text { I value the } \\
\text { time with my } \\
\text { family. }\end{array}$} & Yes $(n=46)$ & $14(100)$ & $32(84)$ & \multirow{2}{*}{$\begin{array}{l}* \\
0.04\end{array}$} & $28(90)$ & $18(86)$ & \multirow[t]{2}{*}{0.61} & $31(84)$ & $15(94)$ & \multirow[t]{2}{*}{0.43} & $\begin{array}{l}16 \\
(80)\end{array}$ & $30(94)$ & \multirow[t]{2}{*}{0.13} & $\begin{array}{l}18 \\
(95)\end{array}$ & $27(84)$ & \multirow[t]{2}{*}{0.27} \\
\hline & No $(n=6)$ & $0(0)$ & $7(16)$ & & $3(10)$ & $4(14)$ & & $6(16)$ & $1(6)$ & & $4(20)$ & $3(6)$ & & $1(5)$ & $6(19)$ & \\
\hline \multirow{2}{*}{$\begin{array}{l}\text { Familial bonds } \\
\text { became } \\
\text { strengthened. }\end{array}$} & Yes $(n=44)$ & $14(100)$ & $30(79)$ & \multirow{2}{*}{$\begin{array}{l}* \\
0.02\end{array}$} & $27(87)$ & $17(81)$ & \multirow[t]{2}{*}{0.55} & $30(81)$ & $14(87)$ & \multirow[t]{2}{*}{0.7} & $\begin{array}{l}15 \\
(75)\end{array}$ & $29(91)$ & 0.13 & $\begin{array}{l}17 \\
\quad(89)\end{array}$ & $27(84)$ & 0.61 \\
\hline & No $(n=8)$ & $0(0)$ & $9(21)$ & & $4(13)$ & $5(19)$ & & $7(19)$ & $2(13)$ & & $5(25)$ & $4(9)$ & & $2(11)$ & $6(19)$ & \\
\hline
\end{tabular}

Chi-square test or Fisher's exact test ${ }^{*} \mathrm{p}<0.05,{ }^{* *} \mathrm{p}<0.01 \mathrm{~N} / \mathrm{A}$ : not applicable Missing value exist in some data

tDo you have anyone who can talk about your illness except family? 
Lastly, regarding the relationship between challenge/hope and QOL/stress-coping ability (Figure 2), there was no relationship with hope. The challenge was significantly related with FACT-G $(r=-0.40, p=0.0015)$ and SOC $(r=-0.40$, $\mathrm{p}=0.0013$ ). In addition, male participants' challenge score (median: 59.5, inter-quartile range: 55.3-63.0) was significantly higher than the woman's score (median: 50.0, inter-quartile range: 47.0-53.0). The challenge of patients who don't have an adviser was significantly higher (median: 50.0, inter-quartile range: 46.5-53.0) than patients with an adviser (median: 59.5, inter-quartile range: 51.5-62.5).
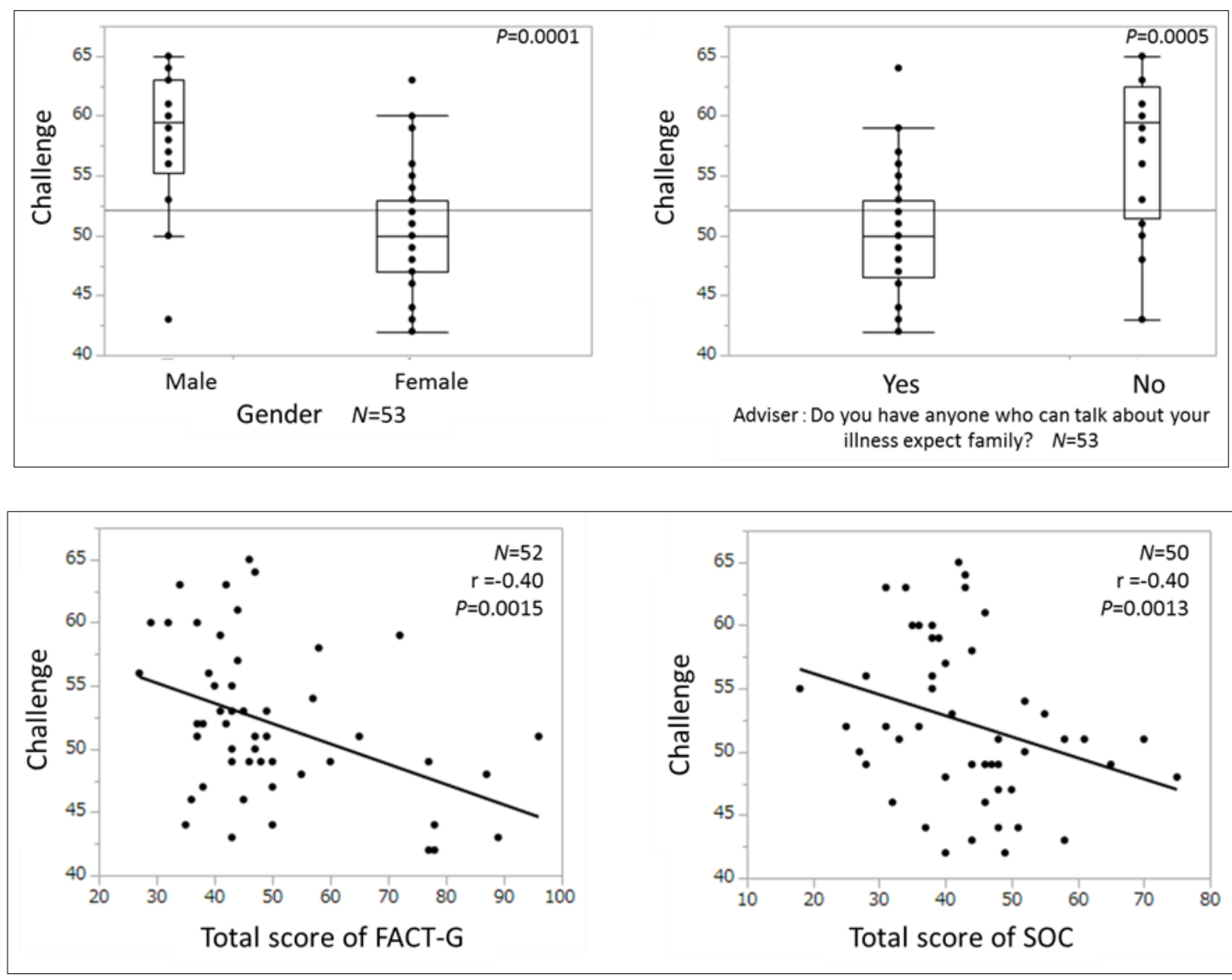

Figure 2 Relationship between challenge and participant's background

\section{Discussion}

\subsection{Summary of result}

This study aimed to clarify 1) the current status of challenge/hope for parental cancer, 2) the factors related to challenge/hope, and 3) the relationship between challenge/hope and QOL/stress-coping ability in the national survey. Participants' QOL and stress-coping ability were low. The main challenges were an inability to fulfill the parental role and children's mental suffering due to loneliness. The main aspects of hope were related to the value of the children's present self, being a parent, and strengthening family ties. Gender, access to an adviser, duration of the disease, and stage of the disease were significantly related to some item of challenge/hope. The total score of challenge was significantly related to gender, access to an adviser, QOL (FACT-G), and SOC.

\subsection{Challenge and hope}

Although parents who have cancer feel a gap between their ideal role/value as a parent and their current status, they derive hope from their relationship with their children. QOL and stress-coping ability are lower than current studies [10-12]. The reason is that this study's sample focused on parents. Health professionals need to support patients to keep their values by providing real-time support in the challenge category. Man feels more challenge, because the parental role is still dominated by the female role in Japan [13]. Because men's decision-making style is different, support methods also need to be different for men. Sufficient social support is crucial for patients to overcome living with cancer, because being a parent is already a tough task. 
The challenge aspect happens with the cancer no matter the stage of the disease $[3,14]$. Patients get a huge shock mentally when they receive the diagnosis; prolonged treatment decreases their performance; even surviving their performance tends not to remain at the same level as before; patients always fear a recurrence; and they have to depend on other people at the end of life.

Hope is a cornerstone of this study since current studies mainly focus on negative aspects like burdens or concerns [2]. Hope lies in the presence of children, which parent's value precious. Parents accumulate experience by fighting back against the aggressive treatment; the more problems they face, the stronger they get. Hope buffers challenge. Support for the children is essential not just for the parents, because by stabilizing the children's mental influences, parents are able to steady their emotions.

\subsection{Imprecation for practice}

A source of support for the family is called CLIMB ${ }^{\circledR}$ (Children's Lives Include Moments of Bravery) program. It was first developed in the United States [15]. The program aims to improve a child's ability to cope with a parent's cancer. Targets are parents who have cancer and their children. In the program, children work on art activities as a group. During the children's session, their parents are able to reduce their own concerns and anxiety related to their children by talking to an adviser in a separate room. Furthermore, communication between parents is also promoted as important of patient's advocacy [16]. Thus, personalized nursing intervention can improve patient compliance and patient's mental state, and worthy of health promotion [17]. Kobayashi suggests that children present post-traumatic stress symptoms and their parents' QOL is improved by using the CLIMB ${ }^{\circledR}$ Program [18].

Parents need to take advantage of available programs, express their feelings and thoughts, look at what they can do, adapt to difficult situations, and acknowledge their own efforts. During periods of unreasonable of physical and mental difficulties, it is necessary that parents talk with healthcare professionals and others. Further studies are needed in order to consider nursing interventions that can reduce challenges of parents during cancer treatment and increase hope.

\section{Limitation}

In Japan, collecting data from multiple institutions is difficult. Nevertheless, the valuable data from a limited number of institutions allowed us to gain a better understanding of challenge and hope in patients who have children. Although the sample size was small due to the minority position of the group, the size was large enough to identify essential topics for further investigation. A father prospective study is necessary truly to explore factors of challenge and hope.

\section{Conclusion}

During cancer treatment, aspects of challenge and hope in patients who have children, do exist. Parents' QOL and stresscoping ability are low. Gender and a prolonged diagnosis period were significantly related with challenge/hope. Challenge was also significantly related with QOL/stress-coping ability.

\section{Compliance with ethical standards}

\section{Acknowledgments}

The authors would like to thank all the participants. We would also like to thank JSPS KAKENHI; Grant-in-Aid for Young Scientists (B) Number 17K17433.

\section{Disclosure of conflict of interest}

The authors declare that they have no competing interests.

\section{Statement of informed consent}

Informed consent was obtained from all individual participants included in the study. 


\section{References}

[1] Krattenmacher T, Kuhne F, Ernst J, Bergelt C, Romer G, Möller B. Parental cancer: factors associated with children's psychosocial adjustment-a systematic review-. Journal of Psychosomatic Research. 2012; 72(5): 344356.

[2] Abe Y, Makabe S, Ito T, Andoh H. Difficulties and Hope for parental cancer: literature review. Health Sciences Bulletin Akita University. 2017; 25(1): 61-69. (In Japanese)

[3] Yoshida K, Kanda K. Relationship between Self-care Agency and Quality of Life among cancer patients undergoing outpatient chemotherapy.pdf. The Kitakanto Medical Journal. 2016; 66: 271-277.

[4] Inoue I, Higashi T, Iwamoto M, Heiney SP, Tamaki T, Osawa K, et al. A national profile of the impact of parental cancer on their children in Japan. Cancer Epidemiol. 2015; 39(6): 838-841.

[5] Surabhi G, Bhupendra SC, Kumari P. Changing pattern of female cancer during last 10 years-experience from a Tertiary Cancer Center. International Journal of Science and Research Archive. 2021; 2(2): 18-27.

[6] Aaron A. The structure and properties of the sense of coherence scale. Social Science \& Medicine. 1993; 36(6): 725-733.

[7] Cella DF, Tulsky DS, Gray G, Sarafian B, Linn E, Bonomi A, et al. The Functional Assessment of Cancer Therapy scale: development and validation of the general measure. Journal of Clinical Oncology. 1993; 11: 570-579.

[8] Shimozuma K. Development and clinical application of QOL scale for patients who have cancer [Internet]. Japan: Nichiisoken; (C) 2001 [cited 2021 Aug 28]. Available from https://www.jmari.med.or.jp/download/WP056.pdf. (In Japanese)

[9] Yanai H, Shigemasu K, Maekawa S, Ichikawa M. In: Yanai H, ed. Factor analysis-theory and methods-. $1^{\text {st }}$ ed Tokyo: Asakura Publishing; 1990; 3-4. (In Japanese)

[10] Drabe N, Klaghofer R, Weidt S, Zwahlen D, Büchi S, Jenewein J. Mutual associations between patients' and partners' depression and quality of life with respect to relationship quality, physical complaints, and sense of coherence in couples coping with cancer. Psychooncology. 2015; 24(4): 442-450.

[11] Driessen CML, de Kleine-Bolt KME, Vingerhoets AJJM, Mols F, Vreugdenhil G. Assessing the impact of chemotherapy-induced peripheral neurotoxicity on the quality of life of cancer patients: the introduction of a new measure. Support Care Cancer. 2012; 20(4): 877-881.

[12] Vilela LD, Allison PJ. An investigation of the correlates of sense of coherence in a sample of Brazilians with head and neck cancer. Oral Oncology. 2010; 46(5): 360-365.

[13] Watanabe Y. Why Does the Gender Gap in Housework Time Remain Wide? The 2015 NHK Japanese Time Use Survey. [Internet]. Japan: NHK Broadcasting Culture Research Institute; (C) 2016 [cited 2021 Aug 28]. Available from https://www.nhk.or.jp/bunken/english/reports/pdf/report_17040601.pdf

[14] Hyphantis T, Goulia P, Zerdes I, Solomou S, Andreoulakis E, Carvalho AF, et al. Sense of Coherence and Defense Style Predict Sleep Difficulties in Early Non-metastatic Colorectal Cancer. Journal of Digestive diseases and sciences. 2016; 61(1): 273-282.

[15] The Children's Treehouse Foundation ${ }^{\circledR}$. CLIMB ${ }^{\circledR}$ Programs [Internet]. State of Colorado: The Children's Treehouse Foundation ${ }^{\circledR}$; (C) 2021 [cited 2021 Aug 28]. Available from https://childrenstreehousefdn.org/climbprograms

[16] Teresa LH, Karen S, Guyanna RA, Megan M, Jennifer RJ. Not the last word: dissemination strategies for patientcentred research in nursing. Journal of Research in Nursing. 2017; 22(5): 388-402.

[17] Yao D, Zhang G, Wang Bo. The Importance and Quality of Personalized Care after Breast Cancer Surgery. Journal of Nursing. 2016; 5(2): 19-21.

[18] Kobayashi M, Heiney SP, Osawa K, Miwa Ozawa M, Matsushima E. Effect of a group intervention for children and their parents who have cancer. Palliat Support Care. 2017; 15(5): 575-586. 